\title{
Crystal structure of betaine dihydrogen selenite, $\mathrm{C}_{5} \mathrm{H}_{13} \mathrm{NO}_{5} \mathrm{Se}$
}

\author{
J. A. Paixão, A. Matos Beja, M. Ramos Silva, L. Alte da Veiga \\ Universidade de Coimbra. Faculdade de Ciências e Tecnologia. Departamento de Física. P-3000 Coimbra, Portugal
}

\section{J. Martín-Gil, F. Martín-Gil}

ETSII. Deparamento de Química Inorgànica, Paseo del Cauce, s/n, SP-47011 Valladolid, Spain

and E. de Matos Gomes

Universidade do Minho. Escola de Ciencias. Departamento de Física. P-4719 Braga Codex, Portugal

Received June 4, 1996, CSD-No. 402502

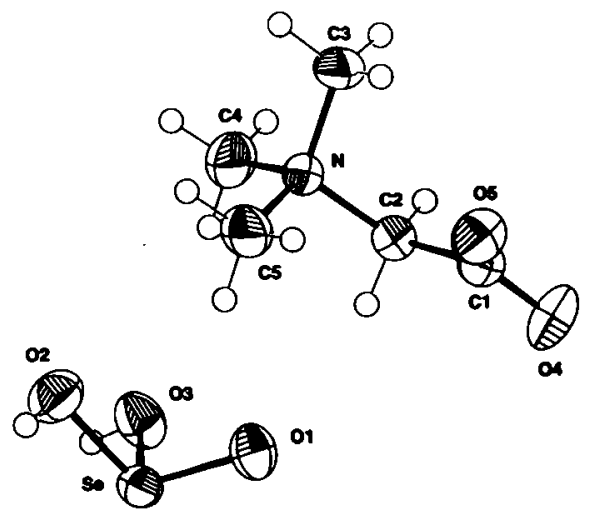

Source of material: The title compound was synthesized by reaction of $1 \mathrm{mmol}$ of betaine (Aldrich, 98\% purity) and $\mathrm{SeO}_{2}$ (Merck, 98\% purity) in a 1:1:1 solution of isopropanol, methanol and water. Crystals were grown from this solution by cooling to $253 \mathrm{~K}$ for a few days.

The $\mathrm{H}_{2} \mathrm{SeO}_{3}$ molecules link the betaine molecules together by hydrogen bonds of $2.626(7) \AA$ between 02 and $04(x-1 / 4$, $-y+3 / 4, z+1 / 4)$ and $2.575(6) \AA$ between 03 and $O 4(x-1 / 4,-$ $y+3 / 4, z-3 / 4$ ). The geometry of the $\mathrm{H}_{2} \mathrm{SeO}_{3}$ molecule (two large and one short $\mathrm{Se}-\mathrm{O}$ bonds) show that the protons have not been transferred to the betaine molecule and thus this compound should be classified as an adduct of the dihydrogen selenite acid. Related compounds: betaine trihydrogen phosphate and betaine trihydrogen arsenate (see ref. 7).

$\mathrm{C}_{5} \mathrm{H}_{13} \mathrm{NO}_{5} \mathrm{Se}$, orthorhombic, Fdd2 (No. 43), $a=28.042(2) \AA$, $b=21.815(2) \AA, c=6.073(2) \AA, V=3715.1 \AA^{3}, Z=16$, $R(F)=0.036, R_{w}\left(F^{2}\right)=0.049$.
Table 1. Parameters used for the $\mathrm{X}$-ray data collection

\begin{tabular}{ll}
\hline Crystal: & colorless transparent prism, \\
& size $0.1 \times 0.1 \times 0.2 \mathrm{~mm}$ \\
Wavelength: & Mo $K \alpha$ radiation $(0.71073 \AA)$ \\
$\mu:$ & $40.30 \mathrm{~cm}^{-1}$ \\
Diffractometer: & Enraf Nonius CAD4 \\
Scan mode: & profile data from $\omega / 2 \theta$ \\
$T_{\text {measurement: }}$ & $293 \mathrm{~K}$ \\
$2 \theta_{\text {max: }}$ & $60^{\circ}$ \\
$\mathrm{N}(\text { hkt })_{\text {unique: }}$ & 1756 \\
Criterion for $I_{0}:$ & $I_{0}>2 \sigma\left(I_{0}\right)$ \\
$N($ param $)$ & 114 \\
Program: & SHELXXL-93: \\
\hline
\end{tabular}

Table 2. Final atomic coordinates and displacement parameters (in $\AA^{2}$ )

\begin{tabular}{llllll}
\hline Alom & Site & $x$ & $y$ & $z$ & $U_{\text {iso }}$ \\
\hline H(2) & $16 a$ & $0.222(2)$ & $0.3695(9)$ & $0.685(7)$ & 0.078 \\
H(3) & $16 a$ & $0.251(1)$ & $0.359(2)$ & $0.133(4)$ & 0.070 \\
H(2A) & $16 a$ & $0.4052(2)$ & $0.4949(2)$ & $0.4959(9)$ & 0.053 \\
H(2B) & $16 a$ & $0.3781(2)$ & $0.4353(2)$ & $0.5634(9)$ & 0.053 \\
H(3A) & $16 a$ & $0.386(1)$ & $0.5674(3)$ & $0.910(3)$ & 0.072 \\
H(3B) & $16 a$ & $0.3405(2)$ & $0.5984(6)$ & $0.812(6)$ & 0.072 \\
H(3C) & $16 a$ & $0.386(1)$ & $0.5913(7)$ & $0.667(2)$ & 0.072 \\
H(4A) & $16 a$ & $0.2900(6)$ & $0.544(1)$ & $0.559(2)$ & 0.082 \\
H(4B) & $16 a$ & $0.307(1)$ & $0.4817(2)$ & $0.458(4)$ & 0.082 \\
H(4C) & $16 a$ & $0.3335(4)$ & $0.543(1)$ & $0.398(2)$ & 0.082 \\
H(SA) & $16 a$ & $0.2967(8)$ & $0.5031(8)$ & $0.928(4)$ & 0.076 \\
H(5B) & $16 a$ & $0.3447(4)$ & $0.474(2)$ & $1.007(3)$ & 0.076 \\
H(5C) & $16 a$ & $0.313(1)$ & $0.4398(7)$ & $0.832(2)$ & 0.076 \\
\hline & & & & &
\end{tabular}

Table 3. Final atomic coordinates and displacement parameters (in $\AA^{2}$ )

\begin{tabular}{|c|c|c|c|c|c|c|c|c|c|c|}
\hline Atom & Site & $x$ & $y$ & $z$ & $U_{11}$ & $U_{22}$ & $U_{33}$ & $U_{12}$ & $U_{13}$ & $U_{23}$ \\
\hline $\mathrm{Se}$ & $16 a$ & $0.27337(2)$ & $0.33236(2)$ & $0.45629(8)$ & $0.0354(2)$ & $0.0302(2)$ & $0.0420(2)$ & $-0.0030(2)$ & $-0.0041(3)$ & $0.0011(3)$ \\
\hline$O(1)$ & $16 a$ & $0.3253(1)$ & $0.3511(1)$ & $0.5523(7)$ & $0.037(2)$ & $0.053(2)$ & $0.062(3)$ & $-0.005(1)$ & $-0.020(2)$ & $-0.006(2)$ \\
\hline $\mathrm{O}(2)$ & $16 a$ & $0.2354(1)$ & $0.3857(1)$ & $0.5807(8)$ & $0.053(2)$ & $0.046(2)$ & $0.057(3)$ & $0.010(2)$ & $0.008(2)$ & $0.000(2)$ \\
\hline$O(3)$ & $16 a$ & $0.2738(1)$ & $0.3706(1)$ & $0.2087(7)$ & $0.045(2)$ & $0.054(2)$ & $0.042(2)$ & $-0.012(2)$ & $-0.006(3)$ & $0.014(2)$ \\
\hline $\mathrm{O}(4)$ & $16 a$ & $0.4629(1)$ & $0.4256(2)$ & $0.688(1)$ & $0.044(2)$ & $0.068(2)$ & $0.045(2)$ & $0.025(2)$ & $-0.002(3)$ & $-0.001(2)$ \\
\hline$O(5)$ & $16 a$ & $0.4297(1)$ & $0.4734(1)$ & $0.9732(8)$ & $0.052(2)$ & $0.052(2)$ & $0.043(3)$ & $0.007(2)$ & $-0.009(3)$ & $-0.006(2)$ \\
\hline $\mathrm{N}$ & $16 a$ & $0.3500(1)$ & $0.5116(1)$ & $0.702(1)$ & $0.028(2)$ & $0.029(2)$ & $0.043(2)$ & $0.001(1)$ & $0.004(3)$ & $0.001(2)$ \\
\hline$C(1)$ & $16 a$ & $0.4305(2)$ & $0.4578(2)$ & $0.779(1)$ & $0.039(3)$ & $0.036(3)$ & $0.038(4)$ & $-0.001(2)$ & $-0.001(3)$ & $0.000(2)$ \\
\hline
\end{tabular}


Table 3. (Continued)

\begin{tabular}{|c|c|c|c|c|c|c|c|c|c|c|}
\hline Atom & Site & $x$ & $y$ & $z$ & $U_{11}$ & $U_{22}$ & $U_{33}$ & $U_{12}$ & $U_{13}$ & $U_{23}$ \\
\hline$C(2)$ & $16 a$ & $0.3910(2)$ & $0.4735(2)$ & $0.6196(9)$ & $0.033(3)$ & $0.034(2)$ & $0.040(3)$ & $-0.001(2)$ & $0.000(3)$ & $-0.001(2)$ \\
\hline$C(3)$ & $16 a$ & $0.3673(2)$ & $0.5726(2)$ & $0.780(1)$ & $0.046(3)$ & $0.029(2)$ & $0.069(5)$ & $-0.002(2)$ & $0.009(3)$ & $-0.010(2)$ \\
\hline C(4) & $16 a$ & $0.3171(2)$ & $0.5208(2)$ & $0.512(1)$ & $0.044(3)$ & $0.053(3)$ & $0.066(6)$ & $0.005(2)$ & $-0.018(3)$ & $-0.002(3)$ \\
\hline$C(5)$ & $16 a$ & $0.3237(2)$ & $0.4791(2)$ & $0.884(1)$ & $0.046(3)$ & $0.047(3)$ & $0.058(5)$ & $-0.005(2)$ & $0.019(3)$ & $0.000(3)$ \\
\hline
\end{tabular}

\section{References}

I. Enraf Nonius: CAD4 Software, Version 5.0. Enraf Nonius, Delft, The Netherlands 1989.

2. Frenz, B. A.: Enraf Nonius SDP-plus Stnucture Determination Package. Version 3.0. Enraf-Nonius, Delft, The Netherlands 1985.

3. Johnson, C. K.: ORTEPII. Report ORNL-5138. Oak Ridge National Laboratory, Tennessee, USA 1976.

4. Main, P.; Fiske, S. J.; Hull, S. E; Lessinger, L.; Germain, G.; Declerq, J.-P.; Woolfson, M. M.: MULTANI1/82. A System of Computer Programs for the Automatic Solution of Crystal Structures from X-ray Diffraction Data. Universities of York, England and Louvain, Belgium 1982.
5. North, A. C. T.; Philips, D. C.: Mathews, F. S.: A semi-empirical method of absorption correction. Acta Crystallogr. A24 (1968) 351-359

6. Sheldrick, G. M.: SHELXL-93, a program for refining crystal structures. University of Göttingen, Germany 1983.

7. Schildkamp, W.; Schäfer, G.; Spilker, J.: The crystal structure of ferroelastic betaine arsenate, $\left(\mathrm{CH}_{3}\right)_{3} \mathrm{NCH}_{2} \mathrm{COO} \cdot \mathrm{H}_{3} \mathrm{AsO}_{4}$, compared with antiferrodistortive betaine phosphate, $\left(\mathrm{CH}_{3}\right)_{3} \mathrm{NCH}_{2} \mathrm{COO} \cdot \mathrm{H}_{3} \mathrm{PO}_{4}$. Z. Kristallogr. 168 (1984) 187-195. 